\title{
Allergieauslöser Kakerlake
}

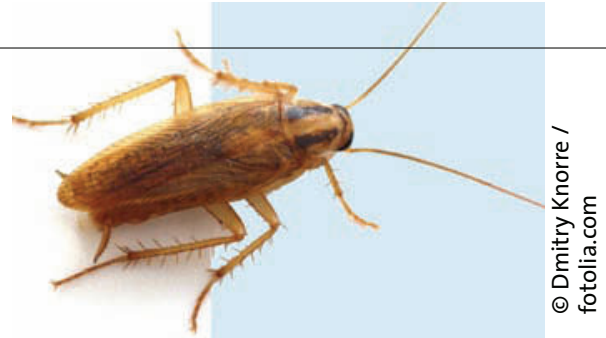

In New York City variiert die Asthmaprävalenz von Kindern je nach Stadtviertel stark: zwischen 3\% und 19\%. Als Ursache vermutet man eine Sensibilisierung gegenüber Kakerlaken und Mäuse. Dieser Vermutung ging man nun in der „New York City Neighborhood Asthma and Allergy"-Studie auf den Grund.

n einer Fall-Kontroll-Studie überprüften Omar Olmedo und sein Team die Hypothese, dass Kinder aus Wohngegenden mit einer hohen Asthmaprävalenz (HAPNs) deutlich stärker Kakerlakenund Mausallergenen ausgesetzt sind als Kinder, die aus Stadtteilen mit niedriger Asthmaprävalenz (LAPNs) stammen. Die Autoren rekrutierten ihre Teilnehmer - 239 Kinder zwischen sieben und acht Jahren und ihre Eltern - über eine New Yorker Krankenkasse, die überwiegend von Menschen mit mittlerem Einkommen genutzt wird. Der allgemeine Gesundheitszustand der Kinder sowie Atemwegsprobleme wurde zu Beginn festgehalten. Außerdem untersuchten die Autoren Hausstaub aus dem Bett der Kinder auf Allergene sowie deren Blut auf spezifische IgE-Antikörper.
Von den 239 Kindern stammten 129 aus HAPNs und 119 aus LAPNs, 128 litten an Asthma und 111 nicht. Im Umfeld der HAPNs-Kinder war die Konzentration der Kakerlaken-, Mausund Katzenhaarallergene deutlich höher als bei LAPNs-Kindern (Bla g 2: p = 0,001; Mus m 1: $\mathrm{p}=0,003$; Fel d 1: $\mathrm{p}=$ 0,003). Bei den Hausstaubmilben-Allergenen verhielt es sich umgekehrt (Der $\mathrm{f} 1$ : $\mathrm{p}=0,001)$. Die Hunde-Allergene wiederum waren in beiden Gruppen in ähnlicher Menge nachweisbar (Can $\mathrm{f} 1: \mathrm{p}=$ 0,54). Dabei zeigte sich: Je höher die Allergen-Exposition, desto höher war auch die Wahrscheinlichkeit einer Sensibilisierung; das galt zumindest für Kakerlaken ( $\mathrm{p}<0,0001)$, Hausstaubmilben $(p=0,009)$ und Katzen $(p=0,001)$, nicht jedoch für Mäuse $(\mathrm{p}=0,58)$ und
Hunde $(p=0,85)$. Der Anteil der Kinder mit spezifischen IgE-Antikörpern gegen die untersuchten Allergene war in beiden Gruppen vergleichbar (LAPNs-Gruppe $53,2 \%$ vs. HAPNs-Gruppe 50,0\%) In HAPNs allerdings gab es deutlich mehr Kinder, die gegenüber Kakerlakenallergenen sensibilisiert waren, als in LAPNs (23,7\% vs. $10,8 \%$; $p=0,011)$. Bei den anderen Allergenen gab es keinen Unterschied zwischen den Gruppen. Mit dem Nachweis entsprechender IgE-Antikörper im Serum stieg die Wahrscheinlichkeit einer Asthmaerkrankung deutlich.

Fazit: Wieder einmal bestätigt sich das enorme allergene Potenzial von Kakerlaken, Hausstaubmilben und Mäusen. Besonders Kakerlakenallergene scheinen Asthma bei Kindern zu forcieren.

Dr. Dagmar Kraus

Olmedo 0 et al. Neighborhood differences in exposure and sensitization to cockroach, mouse, dust mite, and dog allergens in New York. J Alllergy Clin Immunol 2011; 128: 284-92

\section{Anaphylaxie durch Erdnüsse: Wer ist besonders gefährdet?}

\section{Bei Patienten mit Erdnussallergie können schon kleinste Mengen der Hülsenfrucht schwere anaphylaktische Reaktionen hervorrufen. Niederländische Allergologen versuchten jetzt, Faktoren zu finden, mit denen sich gefährdete Kinder und Jugendliche identifizieren lassen.}

E ine aktuelle Studie sollte klären, welche Faktoren dafür verantwortlich sein könnten, dass bei manchen Erdnussallergikern schon eine geringe Menge des Allergens ausreicht, um einen anaphylaktischen Schock auszulösen, andere dagegen weniger sensibel reagieren. Als Maß für diese Sensitivität diente die Reaktionsschwelle der Allergiker im Nahrungsmittelprovokationstest (DBPCFC). Es wurden 126 Kinder in drei Altersgruppen (< 5 Jahre, 5-10 Jahre, > 10 Jahre) mit positivem DBPCFC gegenüber Erdnüssen analysiert.
Schon bei niedrigerer Dosierung waren allergische Reaktionen überdurchschnittlich häufig aufgetreten, wenn die Kinder älter als 10 Jahre waren (Hazard Ratio [HR]: 1,89; 95\%-Konfidenzintervall $[\mathrm{KI}]: 1,28-2,81 ; \mathrm{p}=0,001)$, ihr allergenspezifischer Serum-IgE-Wert $\geq 5,6$ kU/l lag (HR: 2,3; 95\%-KI: 1,37-3,0; $\mathrm{p}<0,0001)$ und dann, wenn keine atopische Dermatitis bestand (HR: 0,45; 95\%-KI: 0,29-0,71; $\mathrm{p}=0,001$ vorhanden vs. nicht vorhanden). Keine signifikanten Zusammenhänge zeigten sich hinsichtlich Geschlecht, familiärer Belastung, einem gleichzeitig bestehenden Asthma oder einer Rhinitis sowie der Stärke früherer allergischer Reaktionen.

Fazit: Diese Studienergebnisse könnten evtl. erklären, so die Wissenschaftler der Universität Groningen, warum Jugendliche häufiger schwere allergische Reaktionen auf Erdnüsse entwickeln als jüngere Kinder. Die Autoren empfehlen, Ernährungsvorgaben bei Allergien strikt einzuhalten sowie älteren Kindern mit Erdnussallergie, die nicht an atopischer Dermatitis leiden und bei denen höhere IgESpiegel gemessen wurden, ein Notfallset mit Epinephrin zur Verfügung zu stellen.

Dr. Christine Starostzik

Van der Zee T et al. The eliciting dose of peanut in double-blind, placebo-controlled food challenges decreases with increasing age an specific IgE in children and young adults. J Allerg Con Immunol 2011; 128: 1031-6 